\title{
Protocol
}

\section{Odor-Taste Learning Assays in Drosophila Larvae}

\author{
Bertram Gerber, Roland Biernacki, and Jeannette Thum
}

The Drosophila larva is an emerging model for studies in behavioral neurogenetics because of its simplicity in terms of cell number. Despite this simplicity, basic features of neuronal organization and key behavior faculties are shared with adult flies and with mammals. Here, we describe a Pavlovian-type learning assay in fruit fly larvae. A group of larvae is sequentially exposed to specific odors in the presence or the absence of sugar, and then tested to determine whether they prefer the odor previously experienced with the reward. The protocol uses a two-group, reciprocal training design: One group of Drosophila larvae is exposed to $n$-amyl acetate (AM) with a sugar reward (+), then subsequently exposed to 1-octanol (OCT) with no reward (denoted AM+/OCT). The other group receives the reciprocal training (AM/OCT + ). The two groups of larvae are then tested for their choices between AM and OCT. Relatively higher preferences for AM after AM+/OCT training than after AM/ OCT + training reflect associative learning and are quantified by the learning index (LI). This method offers a robust, simple, cheap, and reasonably quick test for learning ability (an aversive version is available as well, using either high-concentration salt or quinine as punishment). With the concerted efforts of the Drosophila research community, we anticipate it will allow us to unravel the full circuitry underlying odor-taste learning on a single-cell level.

It is essential that you consult the appropriate Material Safety Data Sheets and your institution's Environmental Health and Safety Office for proper handling of equipment and hazardous materials used in this protocol.

\section{Reagents}

Agarose, $1 \%$ solution in distilled water

Agarose, $1 \%$ solution, supplemented with $2 \mathrm{~m}$ fructose or sucrose

Drosophila larvae

Flies are reared in culture bottles at $25^{\circ} \mathrm{C}$, with a relative atmospheric humidity of $60 \%-70 \%$ and with a $14 / 10-h$ light/dark cycle. Five-day-old larvae are used (90-120 h after egg laying); in particular, only larvae that are still in the food paste, and not larvae that may have begun to crawl up the side of the glass (known as the "wandering stage"), should be used for the learning assay.

n-amyl acetate (AM; Chemical Abstract Services [CAS] 628-63-7)

1-octanol (OCT; CAS 111-87-5)

Paraffin (CAS 8012-95-1)

Adapted from Drosophila Neurobiology (ed. Zhang et al.). CSHL Press, Cold Spring Harbor, NY, USA, 2010. 
B. Gerber et al.

\section{Equipment}

Brush for harvesting larvae

Odor containers

Eight odor containers, a sample of which can be obtained free of charge from the authors, are needed. All containers, instruments, and any item that comes into contact with the containers must always be used for exclusively $A M$ or for exclusively OCT to avoid contamination.

Petri dishes (90-mm)

Petri dish lids, perforated

A sample of the perforated lids can be obtained from the authors free of charge.

Spatula

Stopwatch

\section{METHOD}

The experimental setup is shown in Figure 1.

Preparing the Assay Plates and the Larvae to Be Trained

See Figure 2.

1. On the day before the experiment, prepare eight $90-\mathrm{mm}$ Petri dishes with a thin layer of $1 \%$ agarose only (PUR) and six Petri dishes with 1\% agar containing $2 \mathrm{M}$ fructose (FRU) (alternatively, sucrose can be used). Label the Petri dishes and their lids as PUR or FRU.

Ask a colleague to "code" the Petri dishes for you; for example, the colleague may label the FRUcontaining Petri dishes as " $X$ " and the PUR Petri dishes as "Y." This code should be revealed to the experimenter only after all data have been recorded. Such a procedure ensures that the experimenters' expectations cannot have an impact on how the experiment is performed and/or how data are scored, and should be used when comparing different genotypes.

2. On the morning of the day of the experiment, fill eight odor containers with odorant.

i. Dilute AM 1:50 with paraffin and fill each of four containers with $10 \mu \mathrm{L}$ of the diluted AM.

Diluting AM is important for practical reasons: It ensures that AM and OCT are about equally attractive in experimentally naïve larvae (this may need to be confirmed for your wild-type fly strain of choice), so that learning-induced changes in the relative preference between both odors are easier to detect.

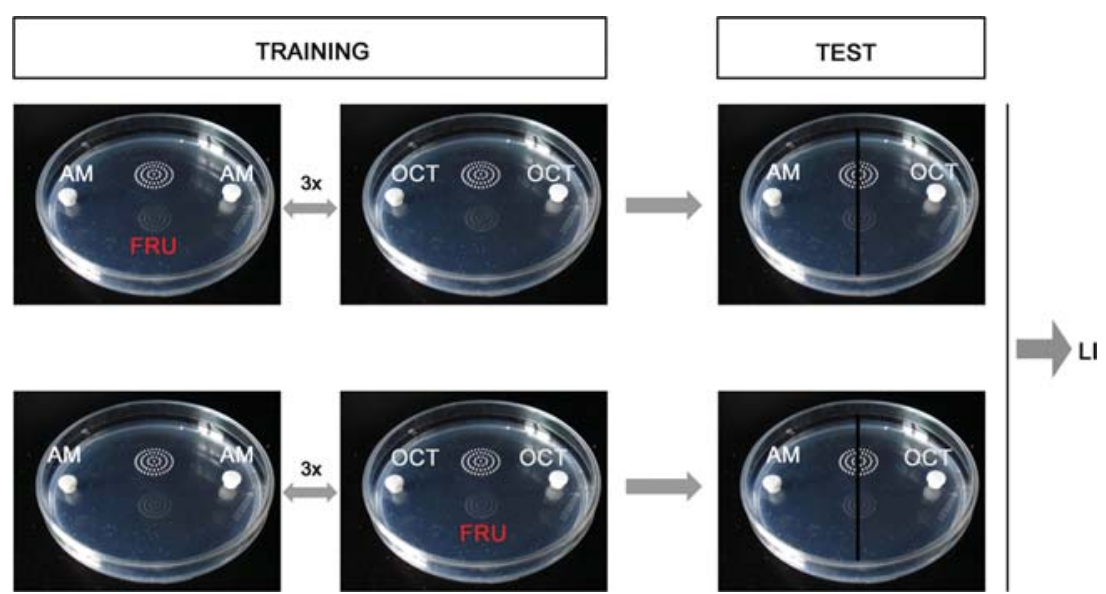

FIGURE 1. The reciprocal principle. A two-group, reciprocal training design is used: In one of the groups, $n$-amyl acetate (AM) is presented with a sugar reward $(+)$ and subsequently 1-octanol (OCT) is presented without reward $(\mathrm{AM}+/ \mathrm{OCT})$. The other group receives reciprocal training (AM/OCT+). After training exposures, animals are tested for their choice between AM and OCT. Relatively higher preferences for AM after AM+/OCT training than after AM/OCT+ training reflect associative learning and are quantified by the learning index (LI). (Image courtsey of B. Michels.) 


\section{Harvesting and Washing of Larvae from the Culture Vials}

3. Using a spatula, remove some food paste containing larvae, and transfer it to a Petri dish with some drops of tap water in it. Wash the larvae by gently stirring.

4. Use a brush to "harvest" 30 larvae, and place them into another Petri dish; then draw the larvae together into a "drop."

It is important that none of the food paste remains within that "drop." Otherwise the food and the food odor may be present during the subsequent training and obscure the learning process.

Training and Testing

The four possible experimental training arrangements are shown in Table 1: AM is presented on the X-Petri dishes and OCT on the $Y$-Petri dishes (arrangements a and b) or, for the reciprocally trained groups, AM is presented on the $Y$ - and OCT on the X-Petri dishes (arrangements $\mathrm{C}$ and $d$ ). With regard to the sequence of training trials, the first trial is performed either with the X-Petri dishes (arrangements a and d) or with the Y-Petri dishes (arrangements $b$ and $c$ ). From our data, the sequence of training trials had no significant effect on test behavior; for example, the larvae distribute between AM and OCT in the same way, regardless of whether they were trained as AM-X first then OCT or OCT first then AM-X. This observation should be confirmed, however, for each respective data set.

5. Carry out the training trial.

i. Using the X-marked Petri dishes, place two AM-filled odor containers opposite one another, $\sim 7 \mathrm{~mm}$ from the edge of the Petri dish.

TABLE 1. The four possible training arrangements

\begin{tabular}{lcccr}
\hline Arrangement & $\mathrm{a}^{\mathrm{a}}$ & $\mathrm{B}$ & $\mathrm{c}$ & $\mathrm{d}$ \\
\hline 1. trial & AM-X & OCT-Y & AM-Y & OCT-X \\
2. trial & OCT-Y & AM-X & OCT-X & AM-Y \\
3. trial & AM-X & OCT-Y & AM-Y & OCT-X \\
4. trial & OCT-Y & AM-X & OCT-X & AM-Y \\
5. trial & AM-X & OCT-Y & AM-Y & OCT-X \\
6. trial & OCT-Y & AM-X & OCT-X & AM-Y \\
TEST & AM vs. OCT & AM vs. OCT & AM vs. OCT & AM vs. OCT \\
\hline
\end{tabular}

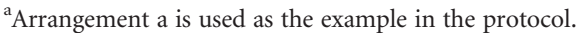


B. Gerber et al.

ii. With the brush, gently place the 30 larvae onto the middle of the X dish. Make sure the larvae are not "trapped" within a drop of water, because they may otherwise have difficulty overcoming the surface tension of the drop. This ensures both that their free wandering on the Petri dish and their ability to smell and/or to taste remains uncompromised.

iii. Place a perforated lid on the dish and start the stopwatch.

The perforation of the lid is meant to improve aeration; to avoid contamination, any given perforated lid should be used exclusively either for AM trials, for OCT trials, or for the test.

iv. At $4 \mathrm{~min}$, prepare the next trial by placing two odor containers filled with OCT into the Y dish. Place a perforated lid on the dish.

v. After a total of $5 \mathrm{~min}$ has passed, transfer the larvae from the $\mathrm{X}$ dish onto the $\mathrm{Y}$ dish and place a fresh perforated lid on the $\mathrm{Y}$ dish. Start the stopwatch and track exposure time for $\mathrm{X}$ min with a stopwatch.

vi. Put the AM containers back in the small Petri dish designated for them, close its lid, and dispose of the used $\mathrm{X}$ dish.

vii. Follow the third to sixth training trials according to Table 1, arrangement a.

6. Carry out the testing.

i. Prepare the test dish during the sixth training trial. At 4 min during the last training trial, place an AM-filled odor container on the left side and an OCT-filled odor container on the right side of the test Petri dish.

To prevent systematic effects of stimuli in the surrounding experimental environment, be sure to carry out the test in one-half of the cases such that AM is presented to the left and OCT to the right (as in this example; see Table 1); in the other one-half of the cases, AM should be placed to the right and OCT to the left (Table 2). This is important in cases in which the larvae can potentially use any directional cues such as a light source (window) for their orientation.

ii. After a total of $5 \mathrm{~min}$ has passed in the last training trial, transfer the larvae from the last $\mathrm{Y}$ dish with the OCT containers onto the test dish. The larvae should be placed in the middle, roughly aligned along the midline; cover the test dish with a perforated lid.

Here also it is important that the larvae are not "trapped" in a drop of water.

iii. Place the two OCT-filled odor containers from the last training dish back into their designated small Petri dish, and close its lid. Dispose of the last training Petri dish.

iv. At 3 min, count the number of animals on the AM side and the number of animals on the OCT side of the dish.

Any animals that at this time point are found on the Petri dish lid are not counted.

v. Put the odor containers back into their respective small Petri dishes, and dispose of the test Petri dish with the larvae in it.

vi. Calculate the PREF score as described in the Data Analysis section (see Discussion).

If you want to finish your experimental work and resume it on the next day, separately wash the AM and the OCT containers. Add odorless detergent to warm water, add the odor containers, and gently

TABLE 2. Versions of the experimental arrangements in which during the test AM is presented to the left and OCT to the right

\begin{tabular}{lcccr}
\hline Arrangement & $\alpha$ & $\beta$ & $\chi$ & \multicolumn{1}{c}{$\delta$} \\
\hline 1. trial & AM-X & OCT-Y & AM-Y & OCT-X \\
2. trial & OCT-Y & AM-X & OCT-X & AM-Y \\
3. trial & AM-X & OCT-Y & AM-Y & OCT-X \\
4. trial & OCT-Y & AM-X & OCT-X & AM-Y \\
5. trial & AM-X & OCT-Y & AM-Y & OCT-X \\
6. trial & OCT-Y & AM-X & OCT-X & AM-Y \\
TEST & OCT vs. AM & OCT vs. AM & OCT vs. AM & OCT vs. AM \\
\hline
\end{tabular}


stir on a magnetic stirrer for at least $1 \mathrm{~h}$. Then, remove the odor containers and dry them separately overnight in an incubator.

Important: Never use the same odor containers for different odors.

7. For the next round of experiments, use a new group of larvae for reciprocal training (e.g., arrangement $\mathrm{c}$ from Table 1) and a fresh set of Petri dishes. That is, in this round, combine AM with the Y-marked and OCT with the X-marked Petri dishes.

i. Remove $\sim 30$ larvae from the culture bottle.

ii. Carry out the training as described in Step 5: The first and second training trials are now $\mathrm{AM}-\mathrm{Y}$ and OCT-X, respectively.

iii. Carry out the test as described in Step 6 and calculate the PREF and LI values as detailed in the Data Analysis section of the Discussion.

iv. Now carry out the experiment according to $\mathrm{b}$, and then according to $\mathrm{d}$ as described in Table 1.

v. Carry out the experiment according to $\alpha, \chi, \beta, \delta$, as described in Table 2 . Ask a colleague to decode the identities of the X- and Y-marked Petri dishes. For the example discussed below, we assume $\mathrm{X}$ corresponds to a sugar-containing Petri dish, and $\mathrm{Y}$ to the Petri dish containing agarose only.

\section{DISCUSSION}

What shall I do? This question is one that confronts humans and animals alike, all the time. To answer it, our needs, sensory impressions, and the possible ways to behave must be adaptively integrated. How this triad of sensory impressions, needs, and behavior is organized is a core scientific problem of neuroscience. As the biological needs of man and animals are in principle similar (to reproduce, to eat, to avoid being eaten, etc.), they provide a reasonable starting point for studying the basic principles of behavior, including its learned alterations. Here, we have described a behavioral tool for such research, focusing on associative odor-taste learning in Drosophila larvae (Scherer et al. 2003, and references below). Studies using odor-electric shock learning (Aceves-Piña and Quinn 1979) are not covered here.

We reasoned that larvae are always hungry, and therefore food should be a particularly powerful reward. A group of larvae is placed onto a sweet-tasting, rewarding sugar substrate $(+)$ and presented with a specific odor A (experience denoted as $\mathrm{A}+$ ). After this exposure, the larvae are transferred to a sugar-free situation, characterized by another odor (B). After three such A+/B experiences, we test the larvae by offering them a choice between the two odors in the absence of sugar. If they have associated odor A with the reward, they should track down this odor in search of sugar. Importantly, this effect is measured relative to reciprocally $(\mathrm{A} / \mathrm{B}+$ ) trained larvae (Fig. 1). Experiments that do not use such a reciprocal design are not considered here; see Gerber and Stocker (2007) for discussion.

Thus, the odor memory promotes the search for sugar. That is, after training with sugar, the test offers the larva a choice with one odor suggesting "over there you will find sugar" and the other "over there you will not find sugar." In the absence of sugar, the larva thus searches for the reward. If sugar actually is present, however, such search is not warranted. Indeed, if the sugar is presented during the test, conditioned behavior is abolished. In contrast, after aversive training (e.g., using quinine as punishment) one odor suggests "over there you will suffer from quinine" whereas the alternative suggests "over there you will not suffer from quinine." In the presence of quinine, therefore, the olfactory quinine associations can give direction to the escape from the aversive reinforcer, whereas if quinine actually is absent, such flight behavior is not warranted. Thus, it is the expected outcome (finding food; escape from unpleasant situations), rather than the "value" of the odor per se, which fuels conditioned behavior (for a more detailed discussion, see Gerber and Hendel 2006). In other 
B. Gerber et al.

words, learned behavior is viewed as an action in pursual of its outcome, rather than a response triggered by the learned odor.

In the Drosophila larva, these kinds of learning are slowly beginning to be understood neurogenetically (see Gerber and Stocker 2007; Gerber et al. 2009; and references therein for more detail): All olfactory sensory neurons originate in the dorsal organ, pass above the pharynx into the brain, and project into the antennal lobe (Fig. 3A,B). Downstream from the antennal lobe, the olfactory pathway bifurcates: One branch of the projection neurons leads to the lateral protocerebrum, comprising premotor centers that support innate olfactory behavior; the other branch takes a "detour" via the mushroom bodies where memory traces for learned odorants can be localized. For learned odor responses, it is via this route that the respective motor centers are thought to be activated. Notably, the lateral protocerebrum thus receives both direct and indirect olfactory input, directly from the projection neurons and indirectly via the mushroom body output neurons, to orchestrate innate and learned behavioral tendencies.

The gustatory pathways, in contrast, originate from multiple external and internal sense organs and run underneath the pharynx, bypass the brain proper, and run toward multiple target areas in the

A

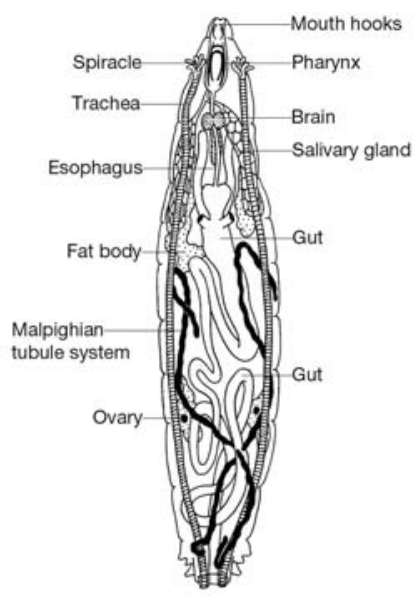

B

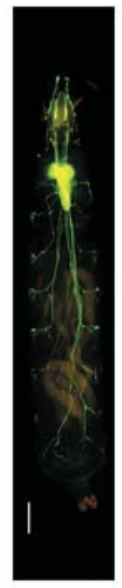

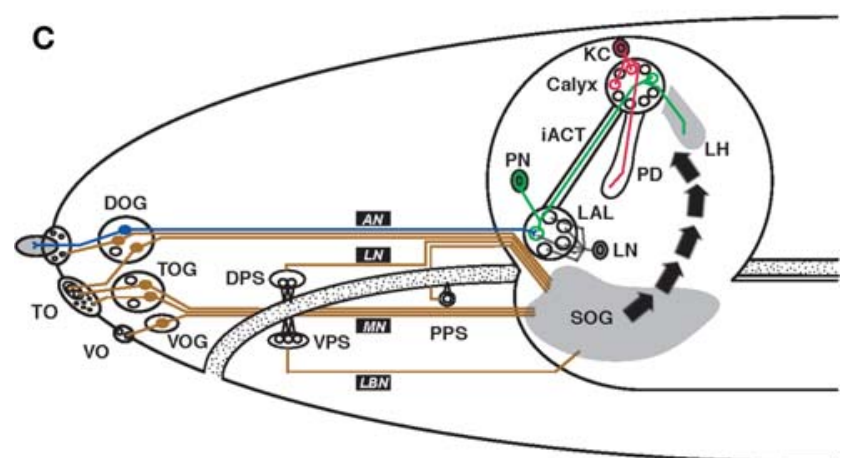

FIGURE 3. (A) Major body parts of a Drosophila larva. (B) Larval nervous system. Fluorescent image of the larval nervous system, clearly showing the brain with the ventral nerve cord and the major nerve cords. Scale bar, $100 \mu \mathrm{m}$. (C) Circuitry. Overview of the chemosensory pathways of the Drosophila larva. Olfactory pathways (blue) project into the brain proper, whereas gustatory input (brown) is collected in various regions of the subesophageal ganglion. The bold arrows indicate the proposed octopaminergic pathway to short circuit a taste-driven "value" signal carried by neurons from the subesophageal ganglion toward the brain. (Reprinted, with permission of Landes Bioscience, from Stocker 2008.) AN, antennal nerve; DO/DOG, dorsal organ/ganglion; DPS, dorsal pharyngeal sense organ; iACT, inner antennocerebral tract; KC, Kenyon cells; LAL, larval antennal lobe; $L B N$, labial nerve; LH, lateral horn; $L N$, local (inter)neurons; $L N$, labral nerve; $M N$, maxillary nerve; PD, pedunculus; PN, projection neuron; PPS, posterior pharyngeal sense organ; SOG, subesophageal ganglion; TO/TOG, terminal organ/ganglion; VO/VOG, ventral organ/ ganglion; VPS, ventral pharyngeal sense organ. $(A)$ Courtesy of The Carnegie Institution; $(B)$ reprinted, with permission, from Sun et al. 1999 (C) National Academy of Sciences, USA); (C) modified, with permission from Landes Bioscience, from Stocker 2008. 
subesophageal ganglion and premotor centers (Fig. 3C). Thus, taste pathways are linked relatively closely to the motor system. Notably, from the subesophageal ganglion, fibers split off from the gustatory pathway to send information about the value of the food to the brain ("good" or "bad": "valuation" neurons).

The mushroom bodies thus bring together the three core aspects of behavioral control: the sensory signal of the odor in terms of the pattern of activated mushroom body cells, the needs of the animal in the form of the "good" signal, and the motor program in the form of the activation of the mushroom body output neurons. Effectively, therefore, within the mushroom bodies a sensory signal ("Which odor?") is reformatted into a motor command ("Go there!") on the basis of the learned "value" of the odor.

In principle the pathways for smelling, tasting, and learning in the larva are similar to those found in adult flies, but in the larvae the numerical simplicity is striking: For example, in the larvae there are only 21 olfactory sensory neurons, 21 antennal lobe glomeruli, $<40$ projection neurons, a not yet fully described small set of local antennal lobe interneurons, a glomerularly defined input region to the mushroom bodies with $<40$ glomeruli, and only $\sim 600$ mushroom body neurons. This numerical simplicity should allow a validation of our admittedly tentative working model of odor-taste learning on the level of single, identified neurons.

\section{Experimental Design}

If you are interested in comparing learning abilities between genotypes, it is important to test for the behavioral specificity of the defect. You therefore need to compare experimentally naïve animals of the different genotypes in terms of (1) their preference between fructose and plain agarose (see Hendel et al. 2005; Niewalda et al. 2008; or Schipanski et al. 2008 for how to do this), (2) their preference between an AM-filled and an empty container, as well as (3) their preference between an OCT-filled and an empty container.

The rationale for not testing the relative preference between the two odors is that, for practical reasons, odor concentrations are chosen such that naive, wild-type animals show $\sim 0$ preference between them; therefore, one may expect both naïve wild-type and naïve mutant larvae to be indifferent with respect to the two odors. This indifference, however, may have different causes in wildtype and mutant larvae: The wild-type may be indifferent, whereas the mutant may be anosmic. This problem is avoided if odor detection, rather than relative preference, is tested.

Still, any mutant learning defect can be seen only after training (i.e., after animals have undergone extensive handling), exposure to the reward, and exposure to the odors. You should therefore test whether a given mutant still is able to (1) detect AM versus an empty odor container if you treat the larvae exactly as during training except that you omit the reward and merely expose to both odors; (2) detect OCT after that same regimen; (3) detect AM versus an empty odor container if you treat the larvae in a training-like way except that you omit the odors and merely expose to the reward; and (4) detect OCT after that same regimen (see Michels et al. 2005). Indeed, handling may stress the animals, change motivation, and/or induce fatigue; repeated odor exposure may lead to sensory adaptation, habituation, and/or latent inhibition and sugar exposure and/or uptake to contextual learning and/or changes in satiety (for discussion, see Gerber and Stocker 2007). Therefore, these kinds of control procedures, introduced in Michels et al. (2005), to us do seem warranted when describing a "learning mutant," and as we anticipate should become standard in the field.

If you wish to extend your analyses to aversive learning (using, e.g., high salt or quinine as punishment), please note that to uncover conditioned aversive behavior one must test in the presence of the respective aversive reinforcer (see above as well as the discussion in Gerber and Hendel 2006).

Finally, one can in principle perform the learning experiment in exactly the same way as detailed here, but omitting one of the two odors from its respective odor container. For example, you can measure the behavior toward AM of larvae that have received paired presentations of AM with the reward during one kind of trial and exposure to an unrewarded Petri dish with an odorless container during the other type of trial (AM+/empty); these larvae then must be compared with larvae that have 
B. Gerber et al.

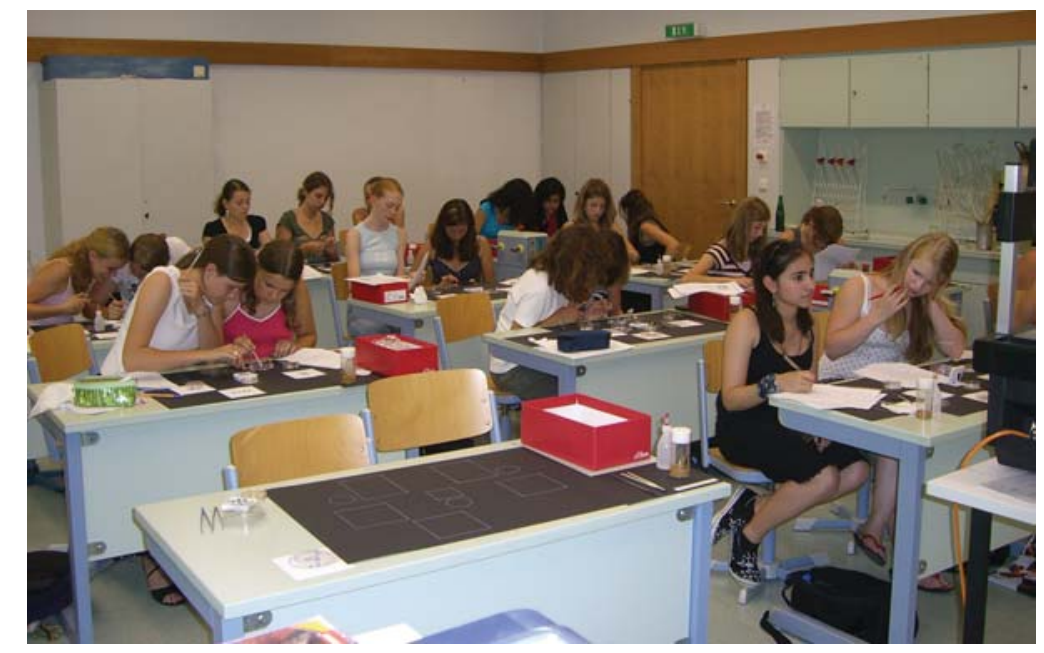

FIGURE 4. Robustness of the assay. The experiment is robust enough for nonacademic settings. Students of the eighth grade at the Gymnasium Stettensches Institut Augsburg are shown performing a one-day course in larval learning.

received unpaired presentations of AM and reward (AM/empty+). Clearly, the test involves an AMfilled container on the one side and an empty container on the other (Selcho et al. 2009).

Note that in an academic setting, experiments are typically performed in a fume hood and without "landmarks" that could allow larval orientation (windows, heaters, etc.). However, these experiments are robust enough to be performed in a nonacademic setting as well-for example, by a group of 20 eighth-grade schoolchildren in a regular classroom (Fig. 4).

Regarding the appetitive learning effect in the example presented here, we want to test whether the joint presentation of an odor with a sugar reward leads to an association between the two. Thus, if larvae are brought to associate different odors with the sugar-as in our case by AM+/OCT training in one case and OCT+/AM training in the other-the larvae should behave differently in the test: Those larvae that were rewarded in the presence of AM should be found more frequently on the AM side relative to those that were rewarded in the presence of OCT. Therefore, the preferences of these two groups are calculated as

$$
\mathrm{PREF}=(\# \mathrm{AM}-\# \mathrm{OCT}) / \# \text { Total }
$$

In this equation, \# indicates the number of larvae observed on the respective half of the test dish. Consequently, a PREF score of 1 means that all the animals were located on the AM side, whereas a PREF score of -1 means that all the animals were found on the OCT side.

These PREF scores are determined for the reciprocally trained groups (arrangements a and c; arrangements $\mathrm{b}$ and $\mathrm{d}$; as well as for $\alpha-\chi$ and $\beta-\delta$ ). They should then be displayed, separated by the odor-sugar contingency (i.e., separately for all AM-rewarded and all OCT-rewarded groups), as boxand-whisker plots with the median as the middle line, the $25 \%$ and $75 \%$ quartiles as box boundaries, and the $10 \%$ and $90 \%$ quantiles as whiskers (Fig. 5A). The median can be determined by dividing an ascending series of the PREF scores by 2 and taking the value right at the midway point, so that $50 \%$ of the PREF scores are larger than this value and 50\% are smaller. Correspondingly, the $25 \%$ quartile marks a PREF value such that $25 \%$ of the PREF scores are larger and $75 \%$ are smaller; the $75 \%$ quartile marks a PREF value such that $75 \%$ of the PREF scores are larger and $25 \%$ smaller. The same logic applies to the $10 \%$ and $90 \%$ quantiles as well. These box-and-whisker plots, rather than a display of the mean and standard error of the mean (SEM), are warranted because typically the kinds of behavioral data from this experiment are not distributed in a Gaussian (bell-shaped) way. 

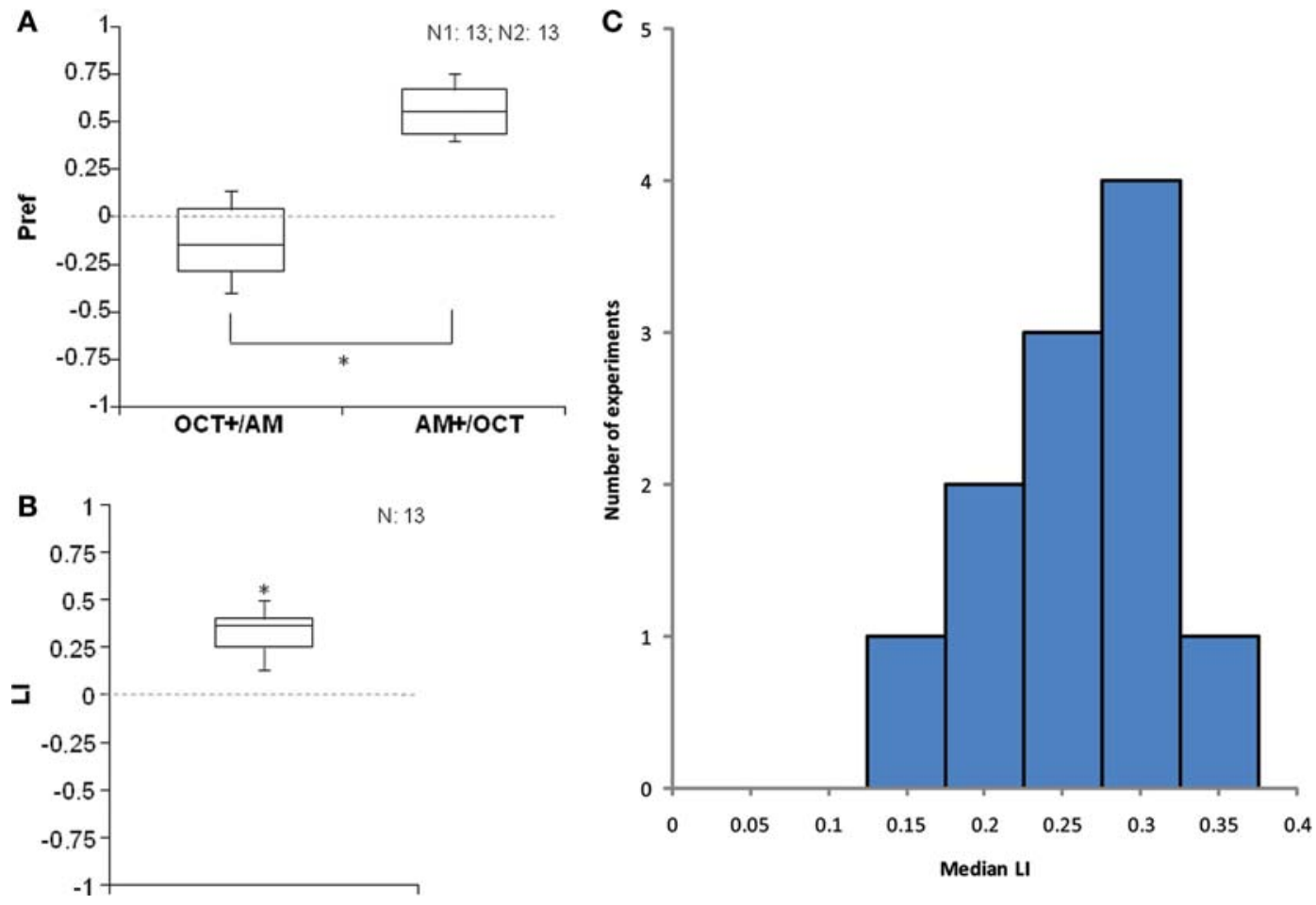

FIGURE 5. Example of results from a nonacademic setting (see Fig. 4). (A) PREF scores of animals rewarded in the presence of OCT, but not of AM (left), or rewarded in a reciprocal manner-in the presence of AM, but not in the presence of OCT (right). Data are presented as median (middle line), and 25\%, 75\% quartiles (box boundaries), and $10 \%, 90 \%$ (whiskers) quantiles. Sample size is 13 for both groups. The asterisk between the plots refers to a MannWhitney U-test. $(B)$ Learning indices (LIs) as calculated from the data in $A$, displayed as box-and-whisker plot. Sample size is 13. (Asterisk) $P<0.05$ in a one-sample sign test. (C) From a series of 11 experiments in various nonacademic settings, the number of experiments finding median learning indices falling into the respective class is presented. Thus, typically the experiments in these kinds of settings usually yield learning indices of between 0.2 and 0.3 .

Our expectation is that the PREF scores of larvae that were rewarded in the presence of AM $\left(\mathrm{PREF}_{\mathrm{AM}+}\right)$ should be higher relative to those that were rewarded in the presence of OCT (PREF $\left.\mathrm{OCT}_{+}\right)$. Note that the comparison between $\mathrm{PREF}_{\mathrm{AM}+}$ and $\mathrm{PREF}_{\mathrm{OCT}+}$ is "buffered" against potential overall preferences for one or the other of the odors. That is, we can conclude that the larvae have learned if either $\mathrm{PREF}_{\mathrm{AM}+}=1$ and $\mathrm{PREF}_{\mathrm{OCT}+}=0$, or if $\mathrm{PREF}_{\mathrm{AM}+}=0.5$ and $\mathrm{PREF}_{\mathrm{OCT}+}=-0.5$ or if $\mathrm{PREF}_{\mathrm{AM}+}=0$ and $\mathrm{PREF}_{\mathrm{OCT}+}=-1$. Whether a learning effect can be shown should be tested by means of a MannWhitney $U$-test comparing the $\mathrm{PREF}_{\mathrm{AM}+}$ versus the $\mathrm{PREF}_{\mathrm{OCT}+}$ values (significance level $5 \%$, i.e., $P<$ $0.05)$. This type of test is warranted because the data are not typically distributed normally, precluding the use of parametric tests such as $t$-tests.

However, we may be interested not only in whether the larvae have learned at all, but also in how much they have learned. This is necessary, for example, for comparing the learning ability of wild-type larvae to that of mutant animals. To this end, we calculate the learning index (Equation 2), to quantify how much the PREF scores of AM-rewarded animals differ from the PREF scores of OCT-rewarded animals (division by 2 ensures that LI scores vary between -1 and 1 ). As we noted above, the sequence of training trials is typically without effect; therefore LIs can be computed either from arrangements a and $\mathrm{c}$ in Table 1 or from arrangements a and $\mathrm{d}$ in Table 1 :

$$
\mathrm{LI}=\left(\mathrm{PREF}_{\mathrm{AM}+}-\mathrm{PREF}_{\mathrm{OCT}+}\right) / 2 .
$$

Thus, LI scores systematically $>0$ show appetitive learning; if the LI scores are $\sim 0$, there is no evidence of learning; if they are systematically negative, one would need to conclude that aversive learning has occurred (for a discussion of the general utility of the LI, see the Appendix to Hendel 
et al. 2005). To display the LI values, a box-and-whisker plot should again be used (Fig. 5B; the median learning indices are shown in Fig. 5C). To test statistically for whether the LI scores differ from 0, a one-sample sign test should be used. This test can be approximated fairly easily: Count how many LI values are $>0$, and how many LI values are $<0$ (e.g., 13 LIs are $>0$, and three LIs are $<0$ ). The lesser of these two numbers is then subtracted from the greater; this difference is known as test statistic $T$ (in our example $T=13-3=10$ ). If $T$ is $>2 \cdot \sqrt{ } n$ (where $n$ is the total number of learning indices different from 0 ), the probability of error is $\langle 5 \%$. In our example, therefore, $2 \cdot \sqrt{ } n=2 \cdot \sqrt{ } 16=8$. Because $T>$ 8 , the LIs across the whole experiment differ significantly from 0 : The larvae have learned. In cases where two genotypes are to be compared in terms of their LI scores, a Mann-Whitney $U$-test is applicable.

In cases of multiple-group comparisons (e.g., when performing rescue experiments or a mutant screen), a Kruskal-Wallis test should be performed first. In case of significance, this should be followed by pairwise $U$-tests that should be properly corrected for such multiple comparisons to keep the experiment-wide error rate at $5 \%$. One such correction, a very conservative one, is known as the Bonferroni correction. This can be performed by dividing the critical $P$ value by the number of pairwise tests performed. Suppose the Kruskal-Wallis test has yielded significance regarding a four-genotype experiment, and you want to do three pairwise comparisons (e.g., wild-type vs. rescue strain, wild-type vs. driver control, and wild-type vs. effector control). Then, the critical $P$ value for each individual U-test should be set as $P<(0.05 / 3)=0.017$, such that the experiment-wide error remains at $P<0.05$.

\section{ACKNOWLEDGMENTS}

The development of this learning paradigm was made possible by the Volkswagen Foundation and the support by R.F. Stocker, Université Fribourg, Switzerland.

B.G. is a Heisenberg Fellow of the Deutsche Forschungsgemeinschaft (DFG), which supports our ongoing research via grants SFB 554 (Arthropod Behavior), SFB TR 58 (Fear, Anxiety, Anxiety Disorders), and GK 1156 (Synaptic and Behavioral Plasticity).

The efforts to make this kind of learning experiment accessible in nonacademic settings were supported by the Robert Bosch Foundation via the Bio-logisch! program. We thank numerous high school and undergraduate students from our laboratory courses, as well as the graduate student teaching assistants advising them, for patience, tireless experimentation, and feedback. All images are courtesy of J. Thum and R. Biernacki, unless noted otherwise.

Translated from the German by R.D.V. Glasgow, Zaragoza, Spain.

We thank J. Wessnitzer and J. Young, University of Edinburgh, for comments.

\section{REFERENCES}

Aceves-Piña EO, Quinn WG. 1979. Learning in normal and mutant Drosophila larvae. Science 206: 93-96.

Gerber B, Hendel T. 2006. Outcome expectations drive learned behaviour in larval Drosophila. Proc Biol Sci 273: 2965-2968.

Gerber B, Stocker RF. 2007. The Drosophila larva as a model for studying chemosensation and chemosensory learning: A review. Chem Senses 32: 65-89.

Gerber B, Stocker RF, Tanimura T, Thum AS. 2009. Smelling, tasting, learning: Drosophila as a study case. Results Probl Cell Differ 47: 139-185.

Heisenberg M, Borst A, Wagner S, Byers D. 1985. Drosophila mushroom body mutants are deficient in olfactory learning. J Neurogenet 2: 1-30.

Hendel T, Michels B, Neuser K, Schipanski A, Kaun K, Sokolowski MB, Marohn F, Michel R, Heisenberg M, Gerber B. 2005. The carrot, not the stick: Appetitive rather than aversive gustatory stimuli support associative olfactory learning in individually assayed Drosophila larvae. $J$ Comp Physiol A 191: 265-279.

Michels B, Diegelmann S, Tanimoto H, Schwenkert I, Buchner E, Gerber B. 2005. A role of synapsin for associative learning: The Drosophila larva as a study case. Learn Mem 12: 224-231.
Neuser K, Husse J, Stock P, Gerber B. 2005. Appetitive olfactory learning in Drosophila larvae: Effects of repetition, reward strength, age, gender, assay type and memory span. Anim Behav 69: 891-898.

Niewalda T, Singhal N, Fiala A, Saumweber T, Wegener S, Gerber B. 2008. Salt processing in larval Drosophila: Choice, feeding, and learning shift from appetitive to aversive in a concentration-dependent way. Chem Senses 33: 685-692.

Scherer S, Stocker RF, Gerber B. 2003. Olfactory learning in individually assayed Drosophila larvae. Learn Mem 10: 217-225.

Schipanski A, Yarali A, Niewalda T, Gerber B. 2008. Behavioral analyses of sugar processing in choice, feeding, and learning in larval Drosophila. Chem Senses 33: 563-573.

Scott K. 2005. Taste recognition: Food for thought. Neuron 48: 455-464.

Selcho M, Pauls D, Han KA, Stocker RF, Thum AS. 2009. The role of dopamine in Drosophila larval classical olfactory conditioning. PLoS One 4: e5897. doi: 10.1371/journal.pone.0005897.

Stocker RF. 1994. The organization of the chemosensory system in Drosophila melanogaster: A review. Cell Tissue Res 275: 3-26. 
Stocker RF. 2001. Drosophila as a focus in olfactory research: Mapping of olfactory sensilla by fine structure, odor specificity, odorant receptor expression, and central connectivity. Microsc Res Tech 55: 284-296.

Stocker RF. 2008. Design of the larval chemosensory system. In Brain development in Drosophila melanogaster (ed. Technau GM), pp. 69-81. Springer, NY.

Strausfeld NJ, Hildebrand JG. 1999. Olfactory systems: Common design, uncommon origins? Curr Opin Neurobiol 9: 634-639.

Sun B, Xu P, Salvaterra PM. 1999. Dynamic visualization of nervous system in live Drosophila. Proc Natl Acad Sci 96: 10438-10443.

Tully T, Cambiazo V, Kruse L. 1994. Memory through metamorphosis in normal and mutant Drosophila. J Neurosci 14: 68-74.
Vosshall LB, Stocker RF. 2007. Molecular architecture of smell and taste in Drosophila. Annu Rev Neurosci 30: 505-533.

Yarali A, Hendel T, Gerber B. 2006. Olfactory learning and behaviour are "insulated" against visual processing in larval Drosophila. J Comp Physiol A 192: 1133-1145.

Zars T. 2000. Behavioral functions of the insect mushroom bodies. Curr Opin Neurobiol 10: 790-795.

Zeng X, Sun M, Liu L, Chen F, Wei L, Xie W. 2007. Neurexin-1 is required for synapse formation and larvae associative learning in Drosophila. FEBS Lett 581: 2509-2516. 


\section{Odor-Taste Learning Assays in Drosophila Larvae}

Bertram Gerber, Roland Biernacki and Jeannette Thum

Cold Spring Harb Protoc; doi: 10.1101/pdb.prot071639

\begin{tabular}{rc}
$\begin{array}{r}\text { Email Alerting } \\
\text { Service }\end{array}$ & Receive free email alerts when new articles cite this article - click here. \\
\hline $\begin{array}{c}\text { Subject } \\
\text { Categories }\end{array}$ & $\begin{array}{c}\text { Browse articles on similar topics from Cold Spring Harbor Protocols. } \\
\text { Behavioral Assays (99 articles) } \\
\text { Drosophila (272 articles) }\end{array}$ \\
\hline
\end{tabular}

